\title{
Sugeno-Type Fuzzy Inference Model for Stock Price Prediction
}

\author{
Uduak A. Umoh \\ Department of Computer Science, University of \\ Uyo, Akwa Ibom State, Nigeria
}

\author{
Alfred A. Udosen \\ Department of Computer Science, University of \\ Uyo, Akwa Ibom State, Nigeria
}

\begin{abstract}
The operations of the prediction of stock price are complex and risky due to fluctuation in the stock market because of the vagueness, incompleteness, and uncertainty of the information used. However, it is therefore as a matter of necessity to seek to foresee stock prices because traders need to know when to invest in order to get the maximum return of the investment. This paper proposes a Sugeno-type fuzzy inference system for stock price prediction using technical indicators as its input values. Knowledge Base, Fuzzification, Inference Engine and Defuzzification are the essential components of our model. We explore Sugeno-type fuzzy inference engine to optimize the estimated result. We evaluate the degree of participation of each input parameter with Trapezoidal membership function. Center of Gravity technique is employed for defuzzification. We employ object oriented design tool to model our database. MATLAB and fuzzy relational database are used in the implementation of our study. The development of this system is based on the selection of stock data history which are studied and used for training the system. This system provides vital support to stock traders, researchers and other financial experts in making decisions as regards stock trading.
\end{abstract}

\section{General Terms}

Soft Computing, Artificial Intelligence, Fuzzy Logic Model, Database management System, Stock Market.

\section{Key Words}

Fuzzy Logic, Stock Price, Technical indicators, Trapezoidal membership function, Object Oriented Tool.

\section{INTRODUCTION}

The operations of the prediction of stock price are complex and risky due to fluctuation in the stock market because of the vagueness, incompleteness, and uncertainty of the information used. Stock trading is the process of buying and selling of stocks based on known signals which can be predicted based on past stock data. Stock price prediction involves the steps taken to determine the future value of a company's stock traded on a financial exchange; making business more profitable as well as making reasonable recommendations as regards stock trading. The accurate predictions of stock price are important for many reasons, chief among these are the need for the investors to hedge against potential market risk and to make profit by trading indexes [1]. Stock is the representation of the ownership in the share of profit, assets, and losses of a company; it is created when a business carves itself into pieces of units called shares and sells them to investors in exchange for cash and stock price is the cost of purchasing or selling a security on an exchange. Predicting stock price has over time been a subject of interest for many financial investors and professional analysts because finding out the appropriate time to sell or buy stock has been a very difficult task as there are too many unknown factors of uncertainty and volatility that have direct influence on stock prices [2] [3].

The prediction of stock price is a highly complicated and very difficult task because there are too many factors such as inflation, short term interest rate, political events, traders' expectations, and environmental factors amongst others that also affect stock price. In addition, stock price series are generally quite noisy, dynamic, nonlinear, complicated, nonparametric, and chaotic by nature [4]. However, the introduction of technology and digital computerization in stock price prediction has paved the way for financial support systems to be developed to make prediction easy. The utilization of intelligent systems such as neural networks, fuzzy logic and genetic algorithms for the purpose of prediction in the field of finance has extensive applications [5]. Several technical indicators are being used in stock price prediction with varying results, they include: Accumulation Distribution Line, Force Index, Money Flow Index, Average True Range, Percentage Price Oscillator, Record High Percentage and Ease of Movement amongst others. Fuzzy logic, artificial neural network, belief calculus, and genetic algorithms amongst others are part of a branch of Artificial Intelligence called soft computing that is essentially used to exploit the tolerance for imprecision, uncertainty, and partial truth in order to achieve tractability, robustness, and low solution cost. [6] defines fuzzy logic as a set of mathematical principles based on degrees of membership rather than classical binary logic that is used for knowledge representation.

This paper proposes Sugeno-type adaptive fuzzy inference system that uses the results of some technical indicators as inputs in combination with the firing strengths of fuzzy rules to make future predictions that can generate buy (low) and sell (high) signals in order to achieve maximum profit. We develop our model using stock data history which is studied and the results computed in the range of predefined limit by a domain expert. We employ MATLAB toolbox and Java programming language and fuzzy relational database to implement our model. The model provides vital support to stock traders, researchers and other financial experts in making decisions as regards stock trading.

Section II presents literature review of the prediction system and the inherent concepts. Section III discusses the analysis of the proposed system IV discusses the design of the model while section V presents the system implementation. Section VI gives a brief conclusion. 


\section{LITERATURE REVIEW}

[7] develop a Takagi-Sugeno-Kang-type fuzzy rule-based system for forecasting Taiwan Stock Exchange price deviation. This model successfully forecasts stock price variation with accuracy close to $97.6 \%$ in TSE index and $98.08 \%$ in MediaTek. [8] develop a hierarchical fuzzy logic system using genetic algorithm to predict the interest rates in Australia. Using a genetic algorithm as a training method for learning the fuzzy rules, the number of rules could be reduced significantly, resulting in more efficient systems. [9] follows a similar approach with a fuzzy inference system, but with other indicators to predict the stock market. The results combining technical analysis and fuzzy logic were very promising. [10] describes a fuzzy based trading system to predict market price movements for investing in portfolio of European, American and Japanese bonds and currency. The system generates a buy or sell signal, but it can also be combined with portfolio allocation mechanisms for automated trading. [11] investigate the current trend of stock price of the Iran Khodro Corporation at Tehran Stock Exchange by utilizing an adaptive fuzzy- neural inference system.

[12] investigate performance analysis of stock price prediction using artificial neural network. The models are used to predict the future stock prices and their performance statistics evaluated. This helps the investor to analyze better in business decisions such as buy or sell a stock. [13], proposes a fuzzy engine model that acts as an expert indicator that can generate buy and sell signals. The fuzzy engine model combines the most popular technical indicators with their firing strengths to provide a new fuzzy indicator that achieves good results compared to the other traditional indicators. Experiments are conducted to evaluate the performance of the model and the results prove that the fuzzy engine model gives more reliable buy and sell positions in different time horizons compared to other technical indicators. [14] examine type-2 fuzzy rule based expert system for stock price analysis. The proposed type-2 fuzzy model applies the technical and fundamental indices as the input variables. The model is tested on stock price prediction of an automotive manufactory in Asia with encouraging results. [15] introduce an intelligent decisionmaking model, based on the application of Fuzzy Logic and Neurofuzzy system (NFs) technology that decide a trading strategy for each day and produce a high profit for each stock. The model is used to capture the knowledge in technical indicators for making decisions such as buy, hold and sell and the experimental results shows higher profits. [16] study fuzzy stock prediction system that integrates the novel computer technologies of stepwise regression analysis (SRA), auto-clustering analysis, recursive least-squares (RLS) and particle swarm optimization (PSO) learning schemes. [17] investigate fuzzy wavelet neural networks for prediction of stock prices. The paper is constructed on the base of a set of TSK fuzzy rules that includes a wavelet function in the consequent part of each rules and train with differential evaluation (DE) algorithm.

[18] Study stock trend prediction system based on Artificial Neural Network (ANN) and fuzzy logic rules using technical indicators and Elliott's wave theory. In this approach the neural network functions as a classifier, where the technical analysis indicators are its input features. The multilayer perceptron (MLP), Support Vector Machine (SVM) and Radial Bases Function (RBF) are tested as classification tools. [19] carry out a probe in a sample of the whole population of the study involves the data and financial record of SAIPA auto-making company which is a member of Iranian stock, for prediction of stock price. The prediction is done using linear and nonlinear models for one ahead and multi ahead in stock price by using exogenous variable of stock market cash index, and the results show the preference of nonlinear neural-Fuzzy model to classic linear model and verify the capabilities of Fuzzy-neural networks in this prediction. [20] investigate analysis on Stock Market Prediction using Data Mining Techniques for evaluation of past stock prices. [21] examine the capability of an ANFIS algorithm to accurately predicting stock market return of the Istanbul Stock Exchange (ISE). The study use six macroeconomic variables and three indices as input variables. The experimental results reveal that the model successfully forecasts the monthly return of ISE National 100 Index with an accuracy rate of $98.3 \%$. ANFIS provides a promising alternative for stock market prediction. [22] study fuzzy rule-based framework for effective control of profitability in a paper recycling plant. [23] present fuzzyneural network model for effective control of profitability in a paper recycling plant. [24] study fuzzy-neural networks approach to model the prediction of stock price. The investigation of neural networks and Autoregressive Integrated Moving Average (ARIMA) models of price data in this research showed unique properties of high precision, quick convergence, strong ability of function approximation, and in operation criteria they showed noticeable superiority in prediction. A fuzzy-neural system to predict financial time series is described by [25]. The prediction of stock and option prices of the S\&P and Dow Jones indices is examined, which results in profitable trading strategies.

\section{ANALYSIS}

The historical stock data for this project is obtained from the Nigerian Stock Exchange, Uyo which is located at Udo Udoma Avenue, Uyo, Akwa Ibom State. Established in 1960 as the Lagos Stock Exchange, it became known as The Nigerian Stock Exchange in December 1977 with about twelve branches having an electronic trading floor each for real-time stock trading through a network of computers connected to a server. The main concern of Nigerian Stock Exchange is to determine the appropriate time to buy, hold or sell stocks. System analysis involves the systematic study of the structure of an adaptive fuzzy neural system model for the prediction of the trends in stock prices. The data collection method, analysis of case study will be explained as well as the architecture of the system design. For this project, stock data history for one year was gathered from interviews and written documents from the Nigerian Stock Exchange in Uyo, Akwa Ibom State. The price lists of daily transactions contains open prices, low prices, high prices, close prices and volume which are equated into different mathematical models called technical indicators that are used for stock prediction. The stock data history collected and used in this system was gotten from Zenith Bank. Stock price prediction is majorly concerned with the use of the history of stock prices gathered in order to tell of its future. The data could be structured, unstructured, static, dynamic, numerical, symbolic, precise or imprecise, certain or uncertain. The system design involves setting of standards to be duly observed during system implementation like variable naming, modularity, coupling, commenting issues, control issues and cohesion in the analysis and design of the system for effective profit optimization. 


\section{DESIGN}

\subsection{SFNISSPP Architecture}

The conceptual architecture of our model is based on [23] and presents in Figure 1. The conceptual architecture comprises the following: The Knowledge Base, Adaptive fuzzy model and user interface.

The knowledge base design of the Sugeno-type fuzzy inference system for stock price prediction comprises of database model and mathematical model. It stores both static and dynamic information about the decision variables. This knowledge base is made up of facts, rules and stock price technical indicators extracted from both structured and unstructured knowledge of experts in the problem domain. The structured knowledge is qualitative while unstructured knowledge is acquired by the stock trading experts through experience.

\subsection{SFNISSPP Database Model}

Figure 2 shows the database model for stock price prediction. SFNISSPP Relationship Diagram is shown in Figure Table 1 shows Company Registration Table with their associated attributes. Table 2 presents Stock Report Table.

COMPANY_REGISTRATION [Company_name collects Company's name, Open price collects stock's open price, Low_price collects stock's close price, High_price collects stock's high price, Close_price, Volume collects stock's volume price] where Company_name is the primary key.

STOCK_REPORT [Technical_indicators collects the mathematical model for the technical indicator used, Recommendation collects the recommended action to take either to Buy, Hold or Sell stock, Date collects the dates of the respective transaction, Company_name collects company's name] where Technical_indicators is the primary key.

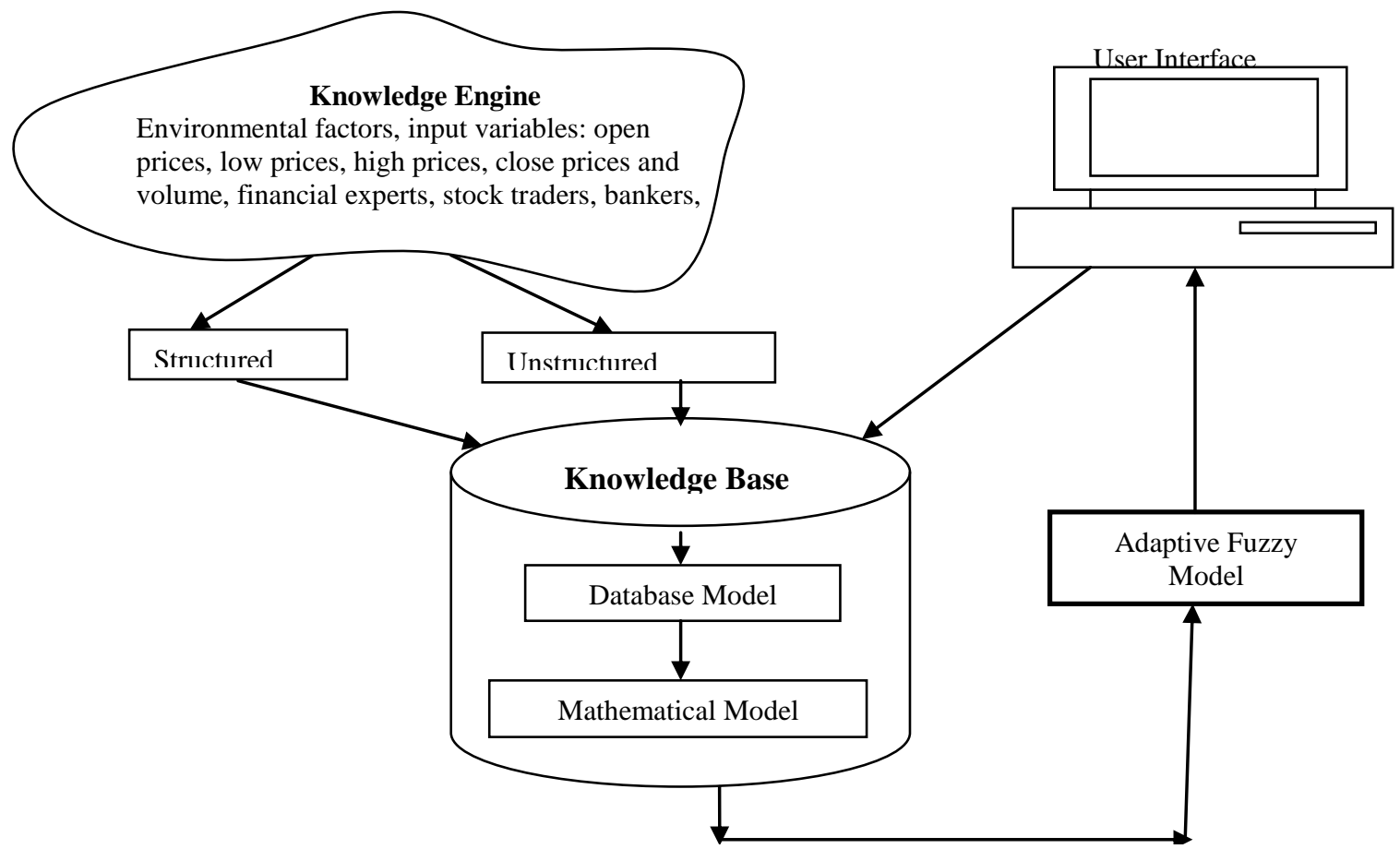

Fig. 1: Adaptive Sugeno Fuzzy System Architecture for Stock Price Prediction

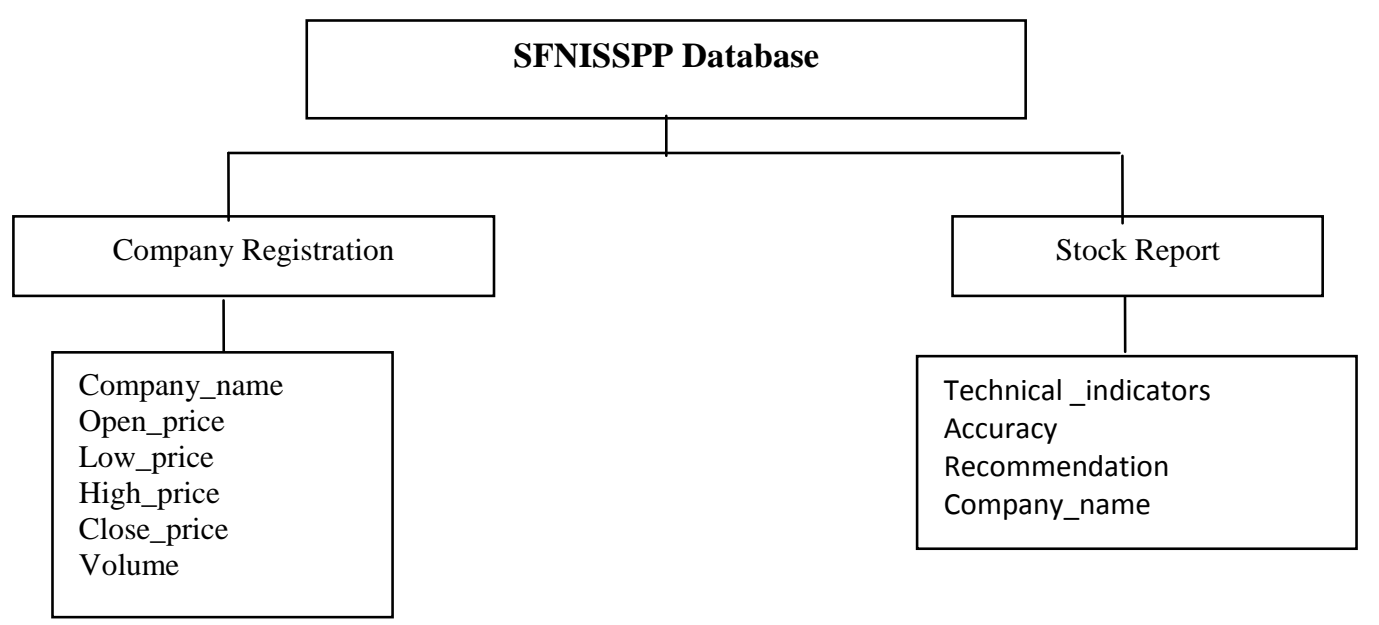

Fig 2 SFNISSPP Database Model for Stock Price Prediction 
Table 1: Company Registration Table

\begin{tabular}{|l|l|l|}
\hline Fields & Data type & Size \\
\hline Date & Char & - \\
\hline Open_price & Float & 6,2 \\
\hline Low_price & Float & 6,2 \\
\hline High_price & Float & 6,2 \\
\hline Close_price & Float & 6,2 \\
\hline Volume & Float & 6,2 \\
\hline
\end{tabular}

Table 2: Stock Report Table

\begin{tabular}{|l|l|l|}
\hline Fields & Data type & Size \\
\hline Technical_indicators & Float & 10,2 \\
\hline Date & Char & - \\
\hline Recommendation & Char & 100 \\
\hline Company_name & Char & 50 \\
\hline
\end{tabular}

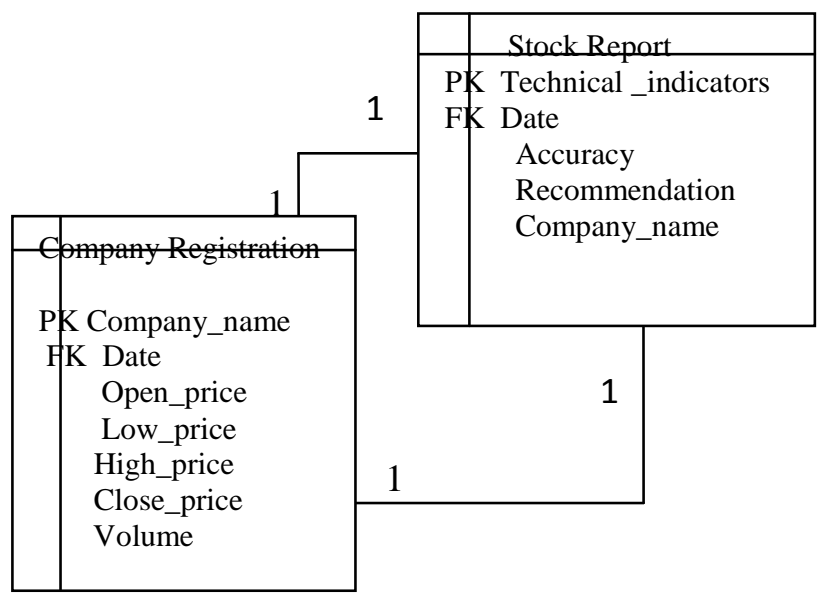

Fig. 3: SFNISSPP Relationship Diagram

\subsection{Mathematical Model}

Technical indicators are used to show price actions (changes in price); they form the input parameters to the developed system. Stock data are collected from the Nigerian Stock Exchange and used for the work as shown in Table 3. The past values of open, low, high and close prices and the volumes of a particular stock are recorded for a sequence of days and stored in database to train the system.

Table 3: Stock price list from the Nigerian Stock Exchange.

\begin{tabular}{|l|l|l|l|l|l|l|}
\hline Y & Date & Open & Low & High & Close & Volume \\
\hline Wed. & 05-Dec & 14.9 & 14.79 & 14.9 & 14.79 & $14,302,790$ \\
\hline Tues. & 04-Dec & 14.9 & 14.8 & 14.91 & 14.81 & $12,141,405$ \\
\hline Mon. & $03-$-Dec & 14.88 & 14.88 & 14.91 & 14.89 & $5,706,231$ \\
\hline Fri. & 30 -Nov & 14.71 & 14.71 & 14.89 & 14.89 & $18,310,264$ \\
\hline Tues. & 16 -Oct & 17 & 17 & 17.13 & 17.01 & $35,780,706$ \\
\hline Mon. & 15 -Oct & 17 & 16.66 & 17 & 16.78 & $16,014,431$ \\
\hline Fri. & $12-$-Oct & 16.94 & 16.94 & 17.01 & 16.95 & $42,499,228$ \\
\hline Thurs. & 11 -Oct & 16.7 & 16.6 & 17 & 16.95 & $17,836,610$ \\
\hline Wed. & $10-$-Oct & 16.54 & 16.21 & 16.99 & 16.71 & $37,314,859$ \\
\hline Tues. & 09-Oct & 15.85 & 15.85 & 17 & 16.87 & $34,615,587$ \\
\hline Mon. & 08-Oct & 15.53 & 15.52 & 15.9 & 15.85 & $18,074,247$ \\
\hline
\end{tabular}

\begin{tabular}{|c|c|c|c|c|c|c|}
\hline Fri. & 05-Oct & 15.49 & 15.15 & 15.6 & 15.52 & $21,162,323$ \\
\hline Thurs. & 04-Oct & 15.1 & 15.1 & 15.53 & 15.55 & $13,059,154$ \\
\hline Wed. & 03 -Oct & 14.99 & 14.97 & 15.05 & 15 & $22,517,867$ \\
\hline Tues. & $02-O c t$ & 14.99 & 14.85 & 15 & 14.96 & $17,180,736$ \\
\hline Fri. & 28-Sep & 14.97 & 14.8 & 15.72 & 14.85 & $42,426,490$ \\
\hline Thurs. & 27-Sep & 14.6 & 14.6 & 14.99 & 14.99 & $19,195,073$ \\
\hline Wed. & 26-Sep & 14.51 & 14.49 & 14.7 & 14.63 & $22,417,338$ \\
\hline Tues. & 25-Sep & 14.51 & 14.5 & 14.56 & 14.55 & $18,349,956$ \\
\hline Mon. & 24-Sep & 14.48 & 14.47 & 14.55 & 14.51 & $23,547,805$ \\
\hline Fri. & 21-Sep & 14.48 & 14.48 & 14.53 & 14.5 & $9,636,587$ \\
\hline Thurs. & 20-Sep & 14.51 & 14 & 14.51 & 14.45 & $19,562,206$ \\
\hline Wed. & 19-Sep & 14.5 & 14.5 & 14.55 & 14.51 & $35,102,272$ \\
\hline Tues. & 18-Sep & 14.48 & 14.34 & 14.53 & 14.5 & $17,288,478$ \\
\hline Mon. & 17-Sep & 14.5 & 14.4 & 14.55 & 14.5 & $67,776,946$ \\
\hline Fri. & 14-Sep & 14.5 & 14.5 & 14.8 & 14.5 & $31,288,044$ \\
\hline Thurs. & 13-Sep & 14.4 & 14.33 & 14.49 & 14.45 & $12,938,303$ \\
\hline Wed. & 12-Sep & 13.9 & 13.82 & 14.5 & 14.41 & $26,745,236$ \\
\hline Tues. & 11-Sep & 14.11 & 13.85 & 14.11 & 13.98 & $24,615,757$ \\
\hline Mon. & 10-Sep & 14.35 & 14.16 & 14.35 & 14.3 & $16,405,985$ \\
\hline Fri. & 07-Sep & 14.15 & 14.11 & 14.81 & 14.31 & $25,347,297$ \\
\hline Thurs. & 06-Sep & 13.95 & 13.81 & 14.35 & 14.11 & $16,281,079$ \\
\hline Wed. & 05-Sep & 13.72 & 13.16 & 13.9 & 13.85 & $32,630,029$ \\
\hline Tues. & 04-Sep & 13.45 & 13.41 & 13.79 & 13.71 & $27,008,561$ \\
\hline Mon. & 03-Sep & 13.4 & 13.4 & 13.52 & 13.46 & $18,229,340$ \\
\hline Fri. & 31-Aug & 13.62 & 13.38 & 13.63 & 13.5 & $28,426,429$ \\
\hline Thurs. & 30-Aug & 13.55 & 13.55 & 13.67 & 13.62 & $26,775,165$ \\
\hline Wed. & 29-Aug & 13.3 & 13.3 & 13.52 & 13.51 & $12,744,752$ \\
\hline Tues. & 28-Aug & 13.06 & 13.06 & 13.27 & 13.25 & $17,988,891$ \\
\hline Mon. & 27-Aug & 13.08 & 13.06 & 13.12 & 13.11 & $17,068,097$ \\
\hline Fri. & 24-Aug & 12.9 & 12.77 & 13.1 & 13.05 & $34,439,317$ \\
\hline Thurs. & 23-Aug & 12.55 & 2.54 & 2.97 & 12.9 & $18,883,885$ \\
\hline Wed. & 22-Aug & 12.52 & 12.5 & 12.55 & 12.52 & $19,437,131$ \\
\hline Fri. & 17-Aug & 12.4 & 12.39 & 12.5 & 12.46 & $16,096,079$ \\
\hline Thurs. & 16-Aug & 12.38 & 12.3 & 12.4 & 12.31 & $10,362,825$ \\
\hline Wed. & 15-Aug & 12.1 & 12.1 & 12.38 & 12.37 & $44,347,013$ \\
\hline Tues. & 14-Aug & 12.1 & 12.1 & 12.13 & 12.1 & $16,811,100$ \\
\hline Mon. & 13-Aug & 12.08 & 12.08 & 12.3 & 12.1 & $29,188,476$ \\
\hline Fri. & 10-Aug & 12 & 12 & 12.09 & 12.07 & $11,402,231$ \\
\hline Thurs. & 09-Aug & 12.16 & 12 & 12.17 & 12 & $16,892,854$ \\
\hline Wed. & 08-Aug & 12.1 & 12.1 & 12.28 & 12.17 & $20,455,699$ \\
\hline Tues. & 07-Aug & 11.82 & 11.62 & 12.2 & 12.1 & $61,871,149$ \\
\hline Mon. & 06-Aug & 11.9 & 11.7 & 11.94 & 11.7 & $4,939,578$ \\
\hline Fri. & 03-Aug & 11.99 & 11.9 & 12.01 & 11.94 & $20,606,478$ \\
\hline Thurs. & 02-Aug & 12 & 12 & 12.01 & 12 & $31,419,030$ \\
\hline
\end{tabular}




\begin{tabular}{|c|c|c|c|c|c|c|}
\hline Wed. & 01-Aug & 11.47 & 11.47 & 11.63 & 11.6 & $8,078,054$ \\
\hline Tues. & 31-Jul & 11.4 & 11.4 & 11.45 & 11.43 & $16,407,752$ \\
\hline Mon. & 30-Jul & 11.45 & 11.4 & 11.5 & 11.4 & $21,747,712$ \\
\hline Fri. & 27-Jul & 11.52 & 11.45 & 11.6 & 11.42 & $9,898,487$ \\
\hline Thurs. & 26-Jul & 11.81 & 11.5 & 11.81 & 11.51 & $16,859,604$ \\
\hline Wed. & 25-Jul & 11.9 & 11.87 & 12.02 & 11.87 & $52,764,435$ \\
\hline Tues. & 24-Jul & 11.8 & 11.8 & 12 & 11.86 & $57,341,109$ \\
\hline Mon. & 23-Jul & 11.84 & 11.76 & 11.9 & 11.82 & $25,756,277$ \\
\hline Fri. & 20-Jul & 11.85 & 11.5 & 11.85 & 11.7 & $19,611,606$ \\
\hline Thurs. & 19-Jul & 12.17 & 11.7 & 12.27 & 11.9 & $38,941,703$ \\
\hline Wed. & 18-Jul & 11.8 & 11.8 & 12.25 & 12.16 & $21,138,375$ \\
\hline Tues. & 17-Jul & 11.57 & 11.56 & 11.79 & 11.73 & $18,860,845$ \\
\hline Mon. & 16-Jul & 11.27 & 11.27 & 11.59 & 11.55 & $12,739,375$ \\
\hline Fri. & 13-Jul & 11.26 & 11.26 & 11.35 & 11.33 & $15,060,058$ \\
\hline Thurs. & 12-Jul & 11.2 & 11.2 & 11.28 & 11.26 & $19,099,134$ \\
\hline Wed. & 11-Jul & 11.3 & 11.2 & 11.3 & 11.2 & $22,118,364$ \\
\hline Tues. & 10-Jul & 11.2 & 11.2 & 11.25 & 11.26 & $17,999,958$ \\
\hline Mon. & 09-Jul & 11.23 & 11.17 & 11.3 & 11.2 & $7,480,203$ \\
\hline Fri. & 06-Jul & 11.16 & 11.16 & 11.26 & 11.26 & $10,199,147$ \\
\hline Thurs. & 05-Jul & 11.05 & 11.05 & 11.25 & 11.15 & $19,152,926$ \\
\hline Wed. & 04-Jul & 11.2 & 11 & 11.3 & 11.01 & $20,658,927$ \\
\hline Tues. & 03-Jul & 11 & 11 & 11.23 & 11.2 & $21,853,101$ \\
\hline Mon. & 02-Jul & 10.9 & 10.9 & 11.05 & 11.02 & $15,391,658$ \\
\hline Fri. & 29-Jun & 10.71 & 10.71 & 11 & 10.91 & $10,283,889$ \\
\hline Thurs. & 28-Jun & 10.78 & 10.6 & 0.82 & 10.76 & $7,076,971$ \\
\hline Wed. & 27-Jun & 10.9 & 10.86 & 10.91 & 10.88 & $10,419,922$ \\
\hline Tues. & 26-Jun & 10.91 & 10.89 & 10.94 & 10.9 & $11,291,494$ \\
\hline Mon. & 25-Jun & 10.9 & 10.78 & 10.92 & 10.91 & $10,419,904$ \\
\hline Fri. & 22-Jun & 10.99 & 10.93 & 11.02 & 10.93 & $10,047,120$ \\
\hline Thurs. & 21-Jun & 11.02 & 10.99 & 11.05 & 10.98 & $17,976,500$ \\
\hline Wed. & 20-Jun & 10.7 & 10.7 & 11.05 & 10.93 & $13,017,139$ \\
\hline Tues. & 19-Jun & 10.52 & 10.52 & 10.65 & 10.68 & $10,615,374$ \\
\hline Mon. & 18-Jun & 10.6 & 10.6 & 10.63 & 10.63 & $14,507,356$ \\
\hline Fri. & 15-Jun & 10.52 & 10.51 & 10.67 & 10.6 & $16,866,915$ \\
\hline Thurs. & 14-Jun & 10.54 & 10.51 & 10.65 & 10.56 & $23,420,226$ \\
\hline Wed. & 13-Jun & 10.5 & 10.5 & 10.65 & 10.6 & $14,361,438$ \\
\hline Tues. & 12-Jun & 10.61 & 10.47 & 10.64 & 10.55 & $21,052,462$ \\
\hline Mon. & 11-Jun & 10.6 & 10.53 & 10.74 & 10.61 & $23,963,459$ \\
\hline Fri. & 08-Jun & 11.31 & 10.75 & 11.31 & 10.8 & $21,423,190$ \\
\hline Thurs. & 07-Jun & 11.35 & 11.3 & 11.43 & 11.3 & $31,087,754$ \\
\hline Wed. & 06-Jun & 11.31 & 11.3 & 11.4 & 11.32 & $30,881,976$ \\
\hline Tues. & 05-Jun & 11.5 & 11.15 & 11.5 & 11.3 & $37,903,273$ \\
\hline Mon. & 04-Jun & 11.5 & 11.5 & 11.6 & 11.51 & $94,103,055$ \\
\hline Fri. & 01-Jun & 11.37 & 11.37 & 11.55 & 11.5 & $27,672,476$ \\
\hline
\end{tabular}

\begin{tabular}{|l|l|l|l|l|l|l|}
\hline Thurs. & 31-May & 11.36 & 11.36 & 11.53 & 11.52 & $30,112,085$ \\
\hline Wed. & 30-May & 11.2 & 11.17 & 11.55 & 11.5 & $37,841,960$ \\
\hline Mon. & 28-May & 11.4 & 11.4 & 11.52 & 11.5 & $20,468,799$ \\
\hline Fri. & 25-May & 11.23 & 11.23 & 11.5 & 11.41 & $18,037,497$ \\
\hline Thurs. & 24-May & 11.3 & 11.2 & 11.4 & 11.2 & $27,548,022$ \\
\hline Wed. & 23-May & 10.91 & 10.9 & 11.35 & 11.29 & $51,201,124$ \\
\hline Tues. & 22-May & 10.55 & 10.55 & 10.95 & 10.9 & $29,881,963$ \\
\hline Mon. & 21-May & 10.74 & 10.54 & 10.74 & 10.6 & $12,294,365$ \\
\hline
\end{tabular}

The details of the parameters used in stock price prediction based on [26] are modified and evaluated as follows:

\subsubsection{Chaikin Money Flow}

This measures the amount of money flow volume over a specific period of time, typically 20 or 21 days. The resulting indicator fluctuates above and below the zero line just like an oscillator to identify changes on money flow. It's presented in (1) - (3).

$$
\begin{gathered}
\text { Money Flow Multiplier }=((C-L)-(H-C)) / \\
(H-L)
\end{gathered}
$$

Money Flow Volume $=M F M *$ Volume

20-period Chaikin Money Flow, $\mathrm{CMF}=$ $\sum_{i=0}^{20}(M F V) / \sum_{1=0}^{20}(V)$

\subsubsection{Force Index}

Force Index is an indicator that uses price and volume to assess the power behind a move or identity possible turning points and is calculated in (4)-(5).

$$
\text { FI }=(C-\text { Prior } C) * \text { Volume }
$$

$$
\text { 13-Period } \mathrm{FI}=\sum_{1=0}^{13}(F I) / 13
$$

\subsubsection{Ease of Movement (EMV)}

The ease of movement (EMV) is a technical indicator that converts the information of the equivolume chart into a numerical equivalent. The calculation is shown in (6)-(9).

$$
\begin{gathered}
\text { Distance Moved }=\left(\frac{H+L}{2}\right)-(\text { Prior } H+ \\
\text { Prior } L) / 2 \\
\text { Box Ratio }=(V / 100,000,000) /(H-L) \\
\text { 1-Period EMV }=\text { Distance Moved } / \text { Box Ratio }
\end{gathered}
$$

14-Period Ease of Movement = 14-Period simple moving average of 1-period EMV $=\sum_{1=0}^{13}(1-$ Period EMV $) / 13(9)$

\subsubsection{Trend Index}

We design Trend Index (TI) model as shown in Figure 5 and (10), after a careful study of the candle sticks used in monitoring the trends in the prices of stocks in the stock market as shown in Figure 5. A number greater than zero indicates a rise in the (positive) trend and a sell of stock is encouraged, a zero indicates a state of no visible change (stagnation point), where traders are advised to halt trading and observe the direction in which the market will follow. Finally, a number below zero shows a fall in the market (negative) trend and a buy of stock is encouraged. 


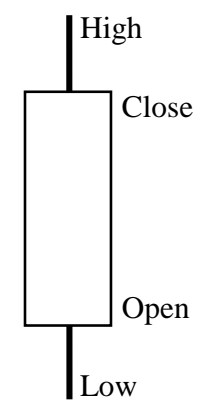

Fig. 4: Stock market candle stick model

14-period $\mathrm{TI}=\sum_{i=0}^{13}\left(\left(\frac{H-I}{C-O}\right) / 13 *\left(\frac{\text { Volume }}{10000000}\right)\right)$

Where $\mathrm{H}=$ High, $\mathrm{C}=$ Close, $\mathrm{O}=$ Open, and $\mathrm{L}=$ Low .

This indicator so developed seems to yield the best of results in stock price prediction based on its computational analysis as compared to the other technical indicators used.

\section{SUGENO-FUZZY INFERENCE} SYSTEM MODEL FOR STOCK PRICE

\section{PREDICTION}

Fuzzy logic model of stock price prediction is presented in Figure 5 .

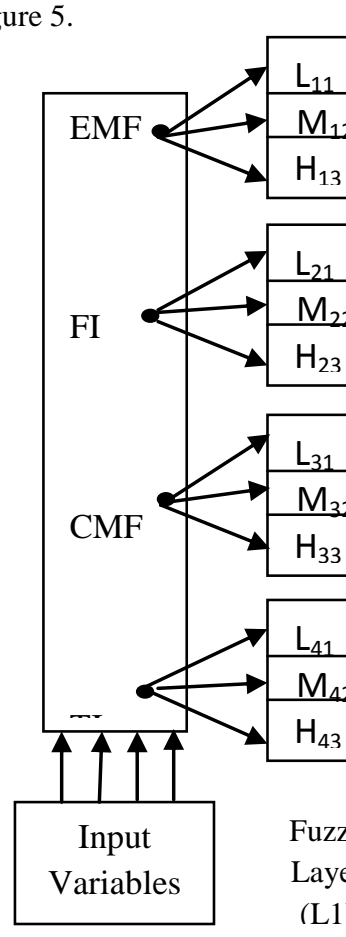

The fuzzy linguistics variables are defined on each input parameter as follows:

Ease of Movement, EMV \{LOW, MEDIUM, HIGH

Force Index, FI \{LOW, MEDIUM, HIGH $\}$

Chaikin Money Flow, CMF \{LOW, MEDIUM, HIGH

Trend Index, TI \{LOW, MEDIUM, HIGH $\}$

Price Movement \{POSITIVE, NO CHANGE, NEGATIVE\}

SFNISSPP universe of discourse is selected for input parameters as; $[-1,1]$ for Chaikin Money Flow, Force Index, Ease of Movement respectively, [-2, 2] for Trend Index and $[0,1]$ for Output parameter.

Layer 1: In this layer, the crisp input values are converted to the fuzzy values by the input MFs. In this paper, we employ Trapezoidal membership functions method in (11) and are evaluated for $\mathrm{i}=(3), \mathrm{j}=(1,2$, and 3$), \mathrm{x}=(\mathrm{EMV}, \mathrm{FI}, \mathrm{CMF}$ and $\mathrm{TI})$ respectively in $(12)-(24)$ as follows: 
where $a i j, b i j, c i j$, and $d i j$ are the premise parameters that characterize the shapes of the input MFs.

\section{Ease of Movement (EMV)}

$$
\begin{aligned}
& \mu_{\text {Low }}(\mathrm{x})= \begin{cases}0 & \text { if } \mathrm{x}<-0.356 \\
0.096(\mathrm{x}+0.356) / 0.0693 & \text { if }-0.356 \leq \mathrm{x} \\
1 & \text { if }-0.287 \leq \mathrm{x}<-0.218 \\
0.267(-0.1487-\mathrm{x}) / 0.0693 \quad \text { if }-0.218<\mathrm{x} & \\
0 & \text { if } \mathrm{x} \geq-0.1487\end{cases} \\
& \mu_{\text {Medium }}\left(\mathrm { x } \left\{\begin{array}{ll}
0 & \text { if } \mathrm{x}<-0.147 \\
(\mathrm{x}+0.147) / 0.0693 & \text { if }-0.147 \leq \mathrm{x}<-0.0777 \\
1 & \text { if }-0.0777 \leq \mathrm{x}<-0.0084 \\
(0.0609-\mathrm{x}) / 0.0693 & \text { if }-0.0084 \leq \mathrm{x}<0.0609 \\
0 & \text { if } \mathrm{x} \geq 0.0609
\end{array}\right.\right. \\
& \mu_{\text {High }}(\mathrm{x})= \begin{cases}0 & \text { if } \mathrm{x}<0.0509 \\
(\mathrm{x}-0.0509) / 0.0693 & \text { if } 0.0509<\mathrm{x}<0.1202 \\
1 & \text { if } 0.1202 \leq \mathrm{x}<0.1895 \\
(0.2588-\mathrm{x}) / 0.0693 & \text { if } 0.1895 \leq \mathrm{x}<0.2588 \\
0 & \text { if } \mathrm{x} \geq 0.2588\end{cases}
\end{aligned}
$$

Force Index (FI)

$$
\mu_{\text {Low }}(x)= \begin{cases}0 & \text { if } x<-0.19231 \\ (x+0.19231) / 0.0348 & \text { if }-0.19231 \leq x<-0.1575 \\ 1 & \text { if }-0.1575 \leq x<-0.1228 \\ (-0.088+x) / 0.0348 & \text { if }-0.1228 \leq x<-0.088 \\ 0 & \text { if } x \geq-0.088\end{cases}
$$$$
\mu_{\text {Medium }}(x)= \begin{cases}0 & \text { if } x<-0.08 \\ (x+0.08) / 0.0348 & \text { if }-0.08 \leq x<-0.05323 \\ 1 & \text { if }-0.05323 \leq x<-0.0185 \\ (0.0163-x) / 0.0348 & \text { if }-0.0185 \leq x<0.0163 \\ 0 & \text { if } x \geq 0.0163\end{cases}
$$$$
\mu_{\mathrm{High}}(\mathrm{x})=\left\{\begin{array}{lc}
0 & \text { if } \mathrm{x}<0.015 \\
(\mathrm{x}-0.015) / 0.0348 & \text { if } 0.015 \leq \mathrm{x}<0.0498 \\
1 & \text { if } 0.0498 \leq \mathrm{x}<0.11934 \\
(0.0154-\mathrm{x}) / 0.0348 & \text { if } 0.11934 \leq \mathrm{x}<0.0154 \\
0 & \text { if } \mathrm{x} \geq 0.0154
\end{array}\right.
$$$$
\mu_{\text {Low }}(x)= \begin{cases}0 & \text { if } x<-0.5035 \\ (x+0.5035) / 0.085 & \text { if }-0.5035 \leq x<-0.4181 \\ 1 & \text { if }-0.4181 \leq x<-0.3326 \\ (-0.2471-x) / 0.085 & \text { if }-0.0185 \leq x<-0.2471 \\ 0 & \text { if } x \geq-0.2471\end{cases}
$$

$$
\begin{aligned}
& \mu_{\text {Medium }}(\mathrm{x})= \begin{cases}0 & \text { if } \mathrm{x}<-07450 \\
(\mathrm{x}+0.2450) / 0.086 & \text { if }-0.2450 \leq \mathrm{x}<-0.1595 \\
1 & \text { if }-0.1595 \leq \mathrm{x}<-0.074 \\
(-0.074-\mathrm{x}) / 0.086 & \text { if }-0.074 \leq \mathrm{x}<0.012 \\
0 & \text { if } \mathrm{x} \geq 0.012\end{cases} \\
& \mu_{\mathrm{High}}(\mathrm{x})= \begin{cases}0 & \text { if } \mathrm{x}<0.01 \\
(\mathrm{x}-0.01) / 0.086 & \text { if } 0.01 \leq \mathrm{x}< \\
1 & \text { if } 0.096 \leq \mathrm{x}<0.181 \\
(0.267-\mathrm{x}) / 0.086 & \text { if } 0.181 \leq \mathrm{x}< \\
0 & \text { if } \mathrm{x} \geq 0.267\end{cases}
\end{aligned}
$$

Trend Index (TI)

$$
\begin{gathered}
\mu_{\text {Low }}(\mathrm{x})= \begin{cases}0 & \text { if } \mathrm{x}<-0.47 \\
(\mathrm{x}+0.47) / 0.102 & \text { if }-0.47 \leq \mathrm{x}<-0.368 \\
1 & \text { if }-0.368 \leq \mathrm{x}<-0.266 \\
(-0.266-\mathrm{x}) / 0.102 & \text { if }-0.266 \leq \mathrm{x}<-0.164 \\
0 & \text { if } \mathrm{x} \geq-0.164\end{cases} \\
\mu_{\text {Medium }}(\mathrm{x})= \begin{cases}0 & \text { if } \mathrm{x}<-0.15 \\
(\mathrm{x}+0.15) / 0.102 & \text { if }-0.15 \leq \mathrm{x}<-0.048 \\
1 & \text { if }-0.048 \leq \mathrm{x}<0.054 \\
(0.156-\mathrm{x}) / 0.102 & \text { if } 0.054 \leq \mathrm{x}<0.156 \\
0 & \text { if } \mathrm{x} \geq 0.156\end{cases} \\
\mu_{\mathrm{High}}(\mathrm{x})= \begin{cases}0 & \text { if } \mathrm{x}<0.146 \\
(\mathrm{x}-\mathrm{0} 146) / 0107 & \text { if } 0146<\mathrm{x}<0) .48 \\
1 & \text { if } 0.248 \leq \mathrm{x}<0.35 \\
(0.452-\mathrm{x}) / 0.102 & \text { if } 0.35 \leq \mathrm{x}<0.452 \\
0 & \text { if } \mathrm{x} \geq 0.452\end{cases}
\end{gathered}
$$

Price Movement (PM)

$\mu_{\text {PriceMovement }}(\mathrm{x})= \begin{cases}0 \text { (Negative) } & \text { if } \mathrm{x}<-1 \\ 0.5 \text { (No Change) } & \text { if }-1 \leq \mathrm{x}<0.5 \\ 1 \text { (Positive) } & \text { if } 0.5 \leq \mathrm{x}<1\end{cases}$

We obtain our rule base from derivation based on the stock history records, expert experience and control engineers. In this paper, the number of MFs for the input variables $E M F$, $F I, C M F$ and $T I$ are determined as 3, 3, 3, and 3, respectively. Each possible combination of inputs and their associated MFs is represented by a rule in the rule base of the Sugeno FIS models. So, the number of rules for FIS models is $81(3 \times 3 \times$ $3 \times 3=81$ ). The application of the Sugeno FIS models to the stock price prediction calculation is given in the following sections. From our knowledge base, 81 rules are defined for the rule base for the decision making unit as shown in Figure 12. 
Fuzzy Rules

1. If ( $E M V$ is $L 11)$ and (FI is $L 21)$ and ( $C M F$ is $L 31)$ and $(F I$ is $L 41)$ then $Z 1=\mathrm{c} 1(E M F, F I, C M F, F I)$

2. If ( $E M V$ is $L 11)$ and ( $F I$ is $L 21)$ and ( $C M F$ is $L 31)$ and ( $T I$ is $M 42$ ) then $Z 2=c 2 E M F, F I, C M F, F I) 3$. if ( $E M F$ is $L 11)$ and $(F I$ is $L 21)$ and $(C M F$ is $L 31)$ and $(T I$ is $H 43)$ then $Z 3=\mathrm{c} 3$ $E M V, F I, C M F, F I)$

4. If ( $E M V$ is $L 11)$ and ( $F I$ is $L 21)$ and ( $C M F$ is $M 32)$ and ( $T I$ is $L 41)$ then $Z 4=c 4 E M F, F I, C M F, F I)$

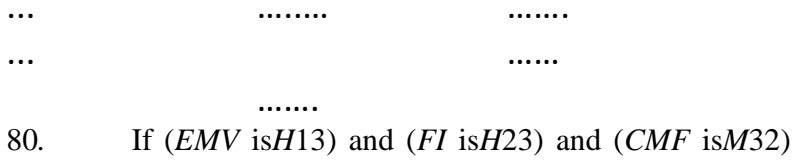
and ( $T I$ is $M 42)$ then $Z 80=\mathrm{c} 53 E M F, F I, C M F, F I)$

81. If ( $E M V$ is $H 13)$ and ( $F I$ isHM23) and ( $C M F$ is $M 32)$ and ( $T I$ is H43) then $Z 81=c 81 E M V, F I, C M F, F I)$

Where,

$Z k=c k=(E M F, F I, C M F, F I) k=1, \ldots, 8 I$

\section{Fig. 12: Fuzzy Rules for Stock price Prediction}

Layer 2: In this layer, the weighting factor (firing strength) of each rule is computed. The weighting factor of each rule, which is expressed as $\alpha k$, is determined by evaluating the membership expressions in the antecedent of the rule. This is accomplished by first converting the input values to fuzzy membership values by using the input MFs in the layer 1 the MAX non-zero minimum value of each of the variables is selected and evaluated using AND function. Only the rules that have strength higher than 0 , would "fire" the output.

Hence, the weighting factors of the rules are computed as follows:

\section{$\lambda_{i n}=$}

$T I_{i 1}\left(v_{0}\right) \wedge E M V_{i 1}\left(x_{0}\right) \wedge F I_{i 1}\left(y_{0}\right) \wedge C M F_{i 1}\left(z_{0}\right), T I_{i 2}\left(v_{0}\right) \wedge E M V_{i 2}\left(x_{0}\right.$ )$\wedge F I_{i 2}\left(y_{0}\right) \wedge C M F_{i 2}\left(z_{0}\right), \ldots, T I_{i n}\left(v_{0}\right) \wedge E M V_{\text {in }}\left(x_{0}\right) \wedge F I_{\text {in }}\left(y_{0}\right.$

)$\wedge C M F_{\text {in }}\left(z_{0}\right)$

Where $\lambda_{\mathrm{i}}$ is the corresponding degree of a given input which satisfies the condition of the ath rule and $i=1,2, . ., 81$.

Layer 3: The normalized weighting factor of each rule, $\bar{\alpha} k$, is computed by using

$$
\bar{\alpha}_{k}=\alpha_{k} / \sum_{i=1}^{\alpha k} \alpha i k=1 \ldots \ldots . .81
$$

Layer 4: In this layer, the output rules can be written as:

$$
\begin{aligned}
& \bar{\alpha} k Z k=\bar{\alpha} k c k(E M V, F I, C M F, T I) \\
& =\bar{\alpha} k\left(E M V_{c k l}+F I_{c k 2}+C M F_{c k 3}+T I_{c k 4}+c k 5\right)
\end{aligned}
$$$$
k=1, \ldots, 81
$$

where $c k$ are the consequent parameters that characterize the shapes of the output MFs. Here, the types of the output MFs (ck) are linear.

Layer 5: In layer 5, defuzzification is performed. The input to the defuzzification process is a fuzzy set (the aggregated output fuzzy set), and the output of the defuzzification process is a single (crisp) number. Here each rule is weighted using it normalized weighting factor and the output of the FIS is computed by the summation of all rule outputs:

\section{Crisp output,}

$$
Z=\sum_{k=1}^{81} \bar{\alpha} k Z k=\left(\sum_{k=1}^{81} \alpha k Z k\right) /\left(\sum_{k=1}^{81} \alpha k\right)
$$

Where $\bar{\alpha}_{\mathrm{i}}$ is a running point in a discrete universe, and $\sum \bar{\alpha} \mathrm{kZk}$ is its membership value in the membership function. The expression can be interpreted as the weighted average of the elements in the support set (Guney, 2009) (Umoh, et al., 2011).

\section{IMPLEMENTATION}

The paper adopts Matlab®/Simulink® and its Fuzzy Logic tool box functions to develop a computer simulation showing the user interface and fuzzy inference to assist the experimental decision for the best control action. From layer 1 , the crisp input values are converted to the fuzzy values by the input MFs. For example, when the fuzzy inputs for EMV, FI, CMF and TI are selected at $-0.1445,0.0156,0.29$ and -0.8 and their corresponding degree of membership evaluated in (29);

EMV $=-0.1445$, Low $=0.06$, Medium $=0.04$, High $=0.0$

$\mathrm{FI}=0.0156$, Low $=0.0$, Medium $=0.02$, High $=0.02$

$\mathrm{CMF}=0.29$, Low $=0.0$, Medium $=0.0$, High $=0.34$

$\mathrm{TI}=-0.8$, Low $=1.0$, Medium $=0.0$, High $=0.0$

The above results indicate that, the degree of membership for an EMV of -0.1445 projects up to the peak function so the result is "Low" membership $=0.06$, "Medium" membership = 0.04 and "High" membership $=0.0$ in the fuzzy sets. Only rules associated with "Low" and "Medium" are actually applied to the output response.

The above results indicate that, the degree of membership for an FI of 0.0156 projects up to the peak function so the result is "Low" membership $=0.0$, "Medium" membership $=0.02$ and "High" membership $=0.02$ in the fuzzy sets. Only rules associated with "Medium" and "High" are actually applied to the output response. The same rule applies to the other input parameters of the membership function.

Fuzzy logic toolbox in Matlab 7.5.0 is utilized for the membership function plots for the EMV, FI, CFM, TI and output (Price Movement) in this paper as shown in Figures 711.

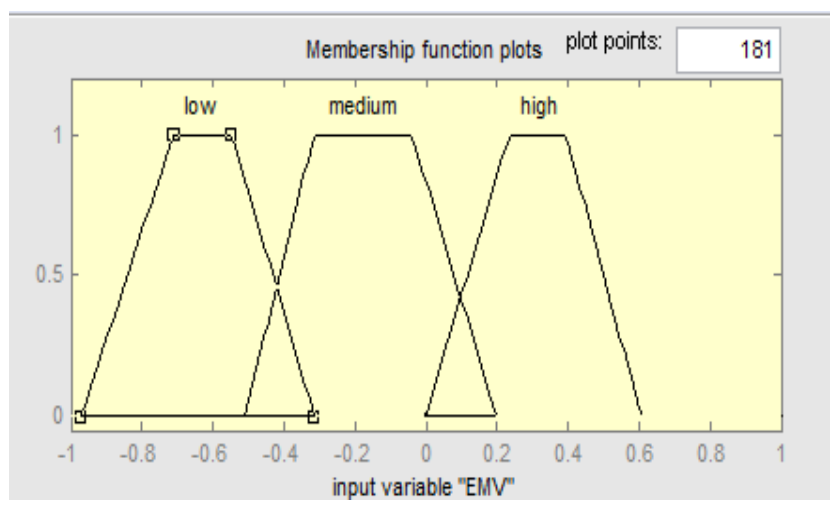

Fig. 7: Membership Function for EMV (Ease of Movement) 


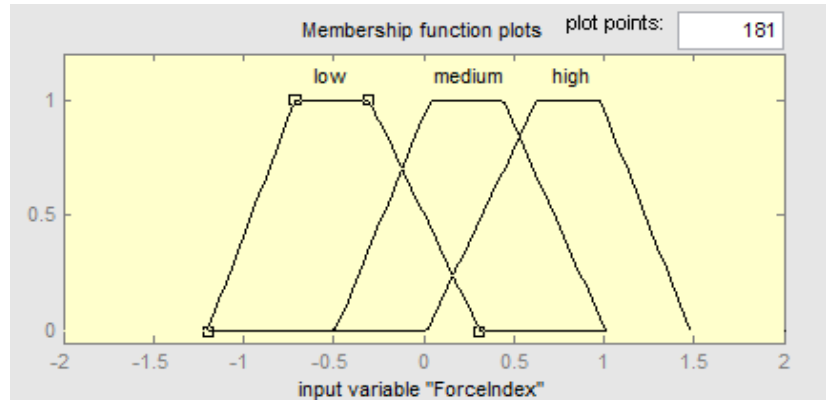

Fig.8: Membership Function for FI (Force Index)

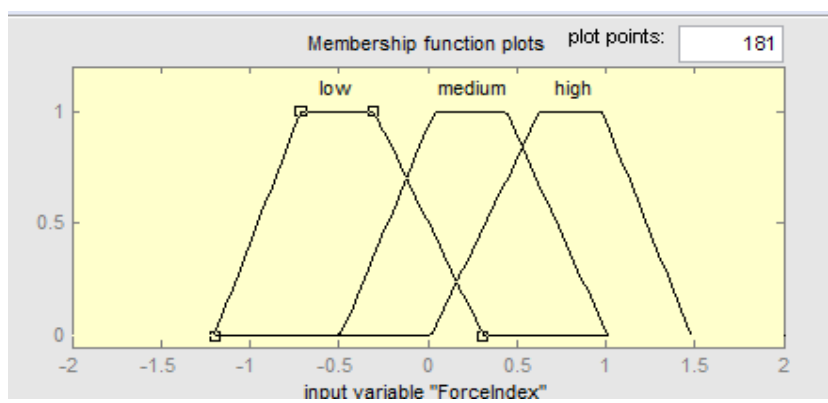

Fig.8: Membership Function for FI (Force Index)

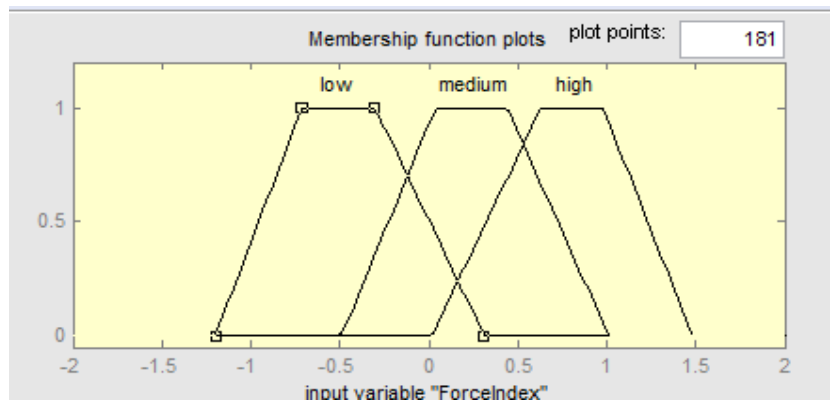

Fig. 9: Membership Function for CMF (Chaikin Money Flow)

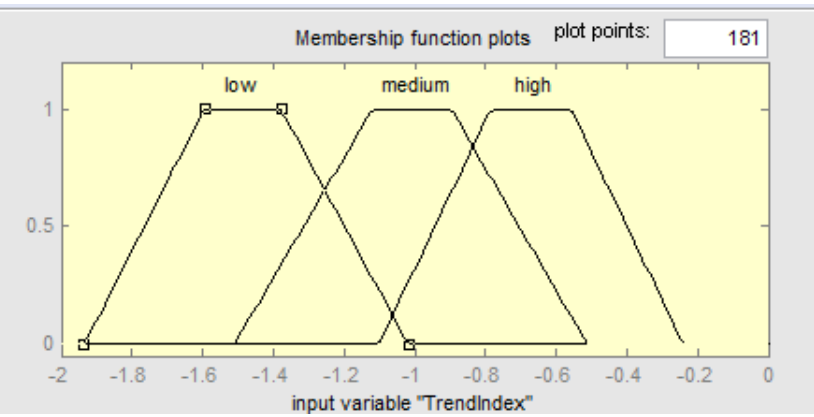

Fig. 10: Membership Function for TI (Trend Index)

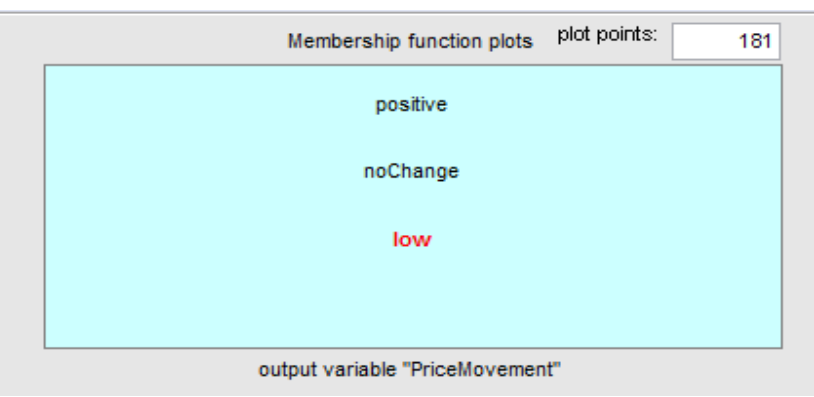

Fig. 11: Linear parameter for Output (PriceMovement)
Results of evaluation of fuzzy rule base inference for four ranges of inputs, EMV, FI, CMF and TI is shown in Tables 3.

Table 3: Rule base evaluation for EMV, FI, CMF and TI at $-0.1445,0.0156,0.29$ and -0.8

\begin{tabular}{|c|c|c|c|c|c|c|}
\hline \multirow{2}{*}{$\begin{array}{l}\text { Rule } \\
\text { Number }\end{array}$} & \multicolumn{4}{|c|}{ Input Variables } & \multirow{2}{*}{ Consequence } & \multirow{2}{*}{$\begin{array}{l}\text { Non zero } \\
\text { Minimum }\end{array}$} \\
\hline & EMV & FI & CMF & TI & & \\
\hline 30 & 0.89 & 0.20 & 0.70 & 1.00 & High & 0.2 \\
\hline 39 & 0.89 & 0.56 & 0.63 & 1.00 & High & 0.56 \\
\hline 46 & $\begin{array}{l}0.99 \\
\end{array}$ & 0.56 & 0.63 & 0.88 & Low & 0.56 \\
\hline 58 & 0.99 & 0.20 & 0.63 & 0.94 & Low & 0.20 \\
\hline 70 & 0.99 & 0.43 & 0.63 & 0.88 & NoC & 0.43 \\
\hline 78 & 0.99 & 0.43 & 0.70 & 0.94 & NoC & 0.43 \\
\hline 80 & 0.89 & 0.20 & $\begin{array}{l}0.77 \\
\end{array}$ & 0.94 & NoC & 0.20 \\
\hline
\end{tabular}

For example, if Rules 30, 39, 46, 58, 70, 78 and 80 fire from the rule base presented in this paper when EMV, FT, CMF and TI values are selected at $-0.1445,0.0156,0.29$ and -0.8 and their corresponding degree of membership evaluated and presented in (29).

From Table 3, the normalized weighting factor of each fired rule, $\bar{\alpha} k$, is computed for High , Low and No change using (26) as follows;

For High, $\alpha_{30}=0.20, \alpha_{39}=0.56$

$$
\bar{\alpha}_{k}=0.56 /(0.20+0.56)=0.7
$$

For Low $, \quad \alpha_{46}=0.56, \alpha_{58}=0.20$

$$
\bar{\alpha}_{k}=0.20 /(0.56+0.20)=0.2
$$

For NoC $\quad \alpha_{70}=0.43, \alpha_{78}=0.43, \alpha_{80}=0.20$

$$
\bar{\alpha}_{k}=0.20 /(0.43+0.43)=0.2
$$

From (27), the output rule values are computed for High, Low and $\mathrm{NoC}$ as;

For High, 0.7 $(-0.1445+0.0156+0.29+(-0.80))=-0.60$

$$
\begin{aligned}
\text { For Low, } & 0.2(-0.1445+0.0156+0.29+(-0.80)) \\
& =-0.20
\end{aligned}
$$

For NoC, $0.2(-0.1445+0.0156+0.29+(-0.80))=-0.20$

From (28), Crisp output, $Z=-0.9$ (Low)

These particular input conditions indicate negative value of 0.90 (Low) therefore low price is expected with 90\% possibility and required system response. This result indicates that the price is low; therefore the investors are advised to buy more stocks.

In Figure 12, having clicked on the Predict menu, rule 59 fired with $\mathrm{EMV}=\mathrm{H}, \mathrm{FI}=\mathrm{L}, \mathrm{CMF}=\mathrm{L}$, and $\mathrm{TI}=\mathrm{M}$ as shown by the scales and the system predicts that the stock price is low. Therefore, recommending the trader to buy more stocks. In Figure 13, having clicked on the Predict menu, rule 74 fired with $\mathrm{EMV}=\mathrm{H}, \mathrm{FI}=\mathrm{H}, \mathrm{CMF}=\mathrm{M}$, and $\mathrm{TI}=\mathrm{M}$ as shown by the scales and the system predicts no change in stock price movement. Therefore, recommending the trader to hold on and observe the direction of the movement of stock prices to determine what action to take next. In Figure 14 as shown below, having clicked on the Predict menu, rule 56 fired with $\mathrm{EMV}=\mathrm{H}, \mathrm{FI}=\mathrm{H}, \mathrm{CMF}=\mathrm{H}$, and $\mathrm{TI}=\mathrm{M}$ as shown by the scales and the system predicts that the stock price is high. Therefore, recommending the trader to sells the available stocks. 


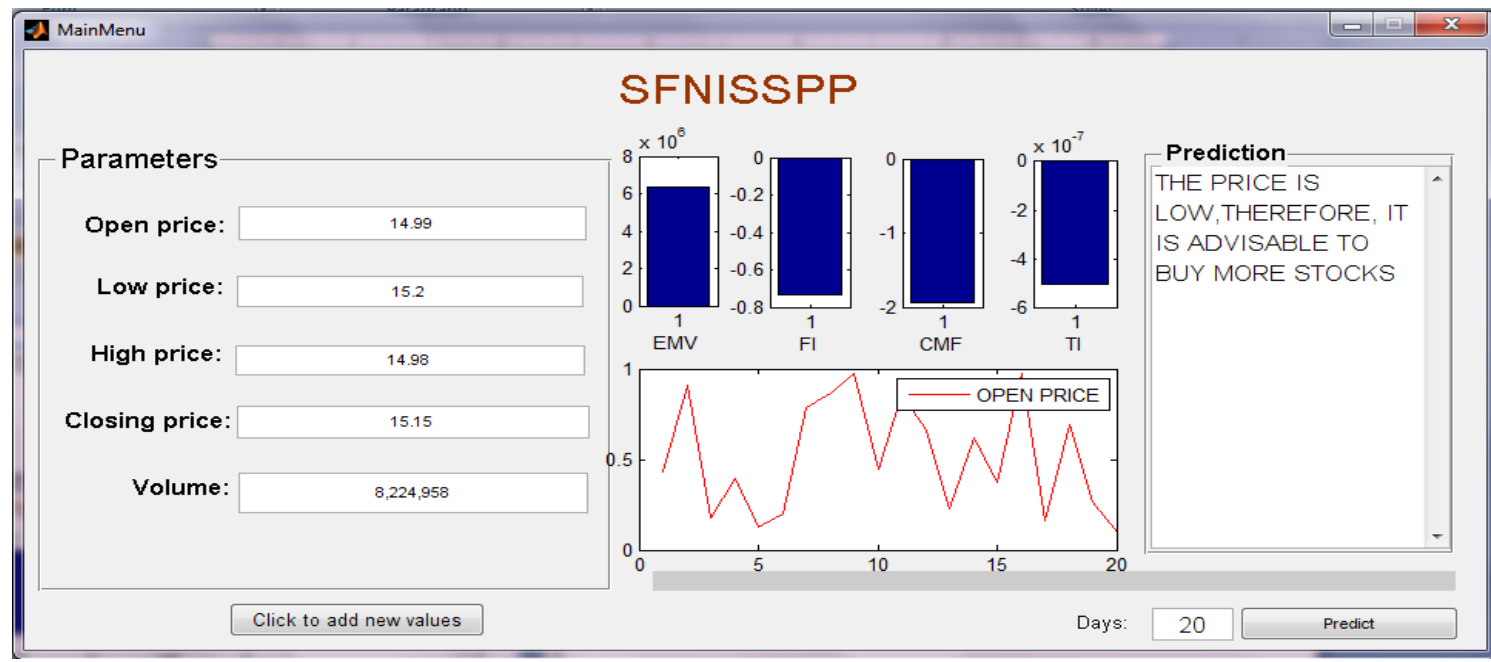

Fig. 12: Main Menu Interface for Low Price

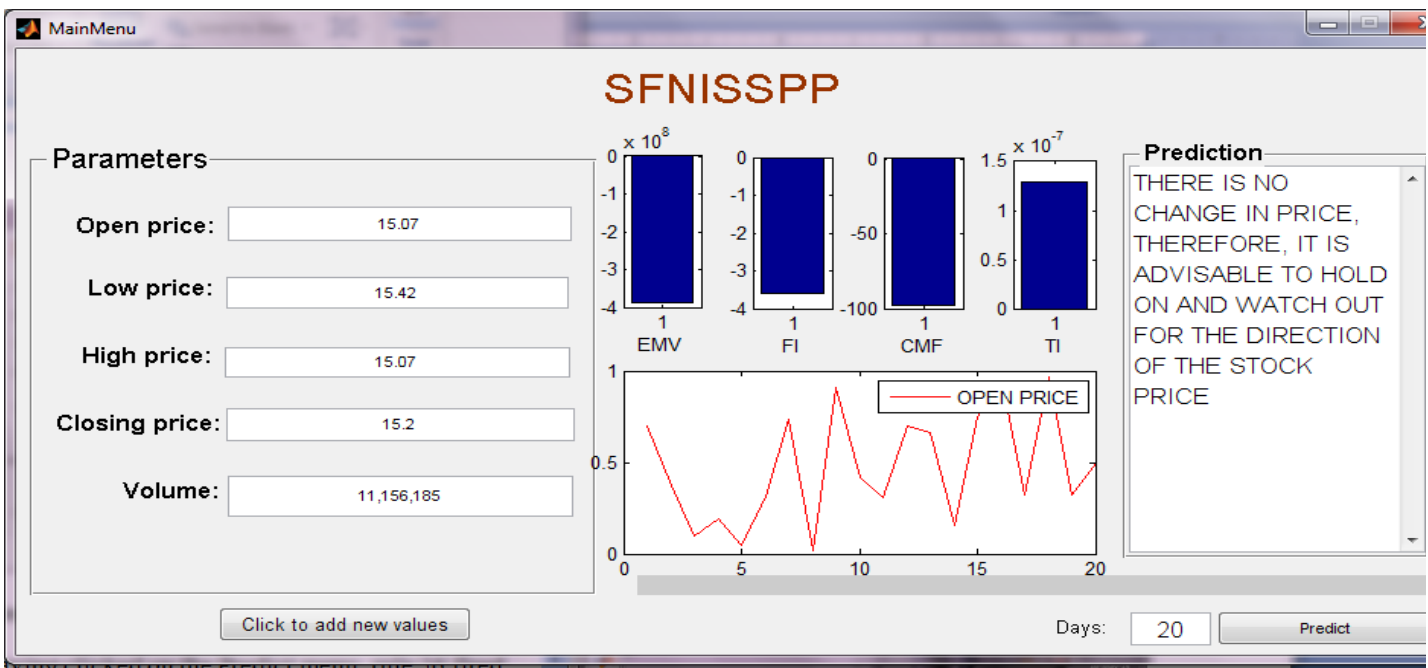

Fig. 13: Main Menu Interface No Change in Price

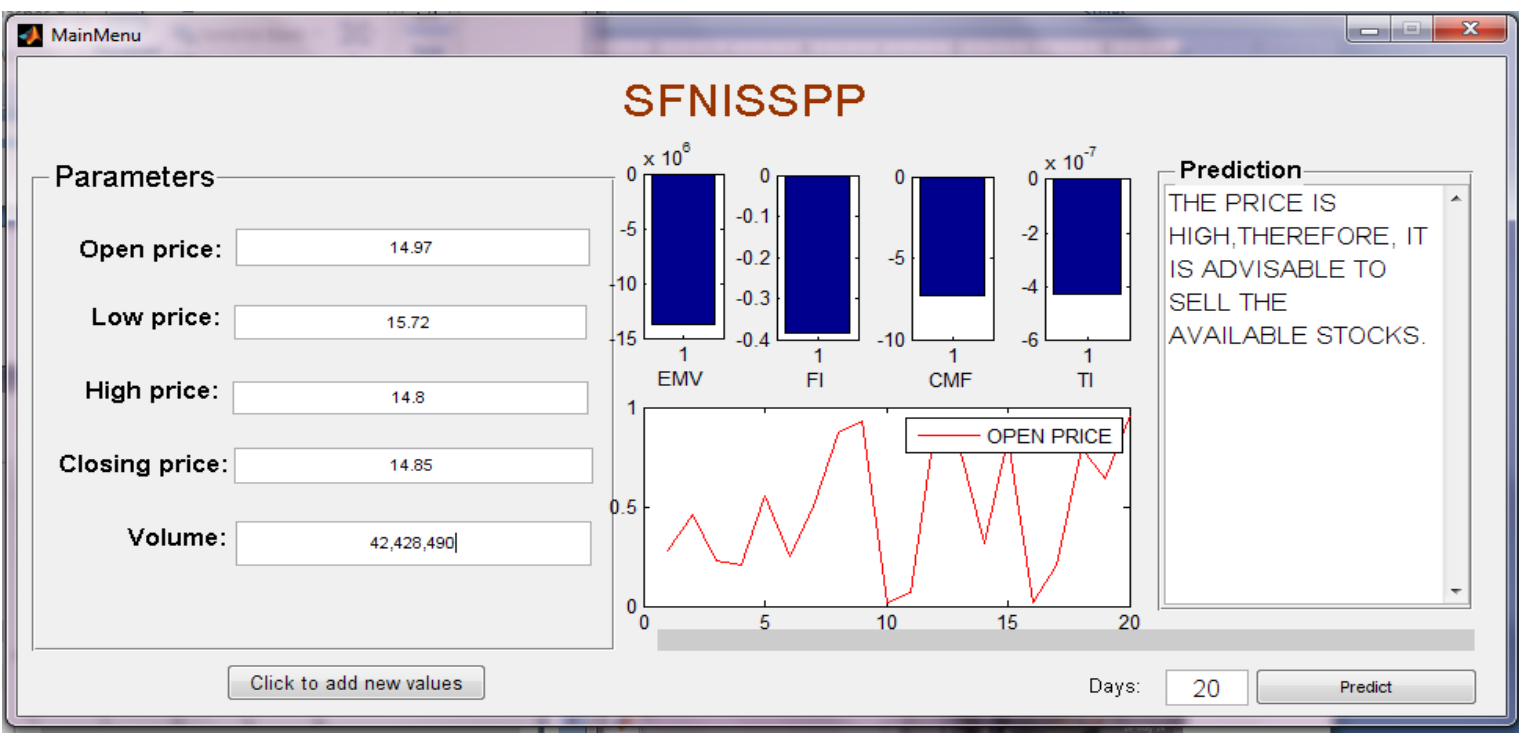

Fig. 14: Main Menu Interface for High Price 


\section{CONCLUSION}

The study constructs a quick and accurate feasible architecture of Sugeno- based fuzzy stock prediction system by applying a linear combination of the significant technical indicators as a consequent to predict the stock price. The proposed system introduced in this paper is tested on different stocks in the historical stock data of the Nigerian Stock Exchange, Uyo which is located at Udo Udoma Avenue, Uyo, Akwa Ibom State. Stock Market for a 3 years period. The system is simulated using Matlab and the results show a good accuracy near $-0.9(90 \%)$ low price with $90 \%$ possibility, indicating that the investors are advised to buy more stocks. At 0.75 (75\%) high price, it shows that the investors are advised to sell stocks while at $0.5(50 \%)$, no change is observed with $50 \%$ possibility. This result indicates that the investors should hold on. The proposed system has the ability to predict the future trend at any trading day. Future work includes detailed analysis of the errors and more advanced methods to overcome these errors. Also, the proposed system is only applied and examined on the Nigerian market stocks. The system can explore other stock market. In addition, the system can be optimized by exploring neural network tool.

\section{REFERENCES}

[1] Ching, L. S., Jyh C., and Shih, M. Y. (2010). International Journal of Education and Information Technologies, 4(3).

[2] Nassim, H. and Ali, A. (2011). Stock price prediction using a fusion model of wavelet, fuzzy logic and ANN. International Conference on E-business, Management and Economics.IPEDR, 25. IACSIT Press, Singapore.

[3] Hemanth, K. P, Prashanth, K. B., Nirmala, T. V., Basavaraj S. P. (2012). Neuro Fuzzy based Techniques for Predicting Stock Trends. IJCSI International Journal of Computer Science 9, 4(3), 1694-0814.

[4] Yudong, Z., \& Lenan, W. (2009). Stock market prediction of S\&P 500 via combination of improved BCO approach and BP neural network. Expert Systems with Applications, 36(5), 8849-8854.

[5] Boyacioglu, M. A. \& Avci, D. (2010). An Adaptive Network-Based Fuzzy Inference System (ANFIS) for the prediction of stock market return: The case of the Istanbul Stock Exchange. Expert Systems with Applications 37. 7908-7912.

[6] Zadeh, L. A. (1965). Fuzzy Sets. Information and Control 8, 338-353.

[7] Chang, P. C., \& Liu, C. H. (2008). A TSK type fuzzy rule based system for stock price prediction. Expert Systems with Applications, 34(1), 135-144.

[8] Mohammadian, M. and Kingham, M. (2004). An adaptive hierarchical fuzzy logic system for modelling of financial systems. International Journal of Intelligent Systems in Accounting, Finance and Management, 12(1), 61-82.

[9] Hiemstra, Y. (1994). A stock market forecasting support system based on fuzzy logic. In Proceedings of the Twenty-Seventh Hawaii International Conference on System Sciences, 3, 281-287.
[10] Cheung, W. M. and Kaymak, U. (2012). A Fuzzy Logic Based Trading System. Ching, L. S., Jyh C., and Shih, M. Y. (2010). International Journal of Education and Information Technologies. 3, 4.

[11] Abbasi, E. \& Abouec, A. (2008). Stock price forecast by using neuro-fuzzy inference system. Proceedings of World Academy of Science, Engineering and Technology, 36, 320-323.

[12] Sureshkumar, K. K. \& Elango, N. M. (2012). Performance Analysis of Stock Price Prediction using Artificial Neural Network, Global Journal of Computer Science and Technology (GJCST), 12(1), 2-9.

[13] Ahmad, S. M, Gayar, N, Elazim, H. A (2006). A Fuzzy Engine Model for Efficient Stock Market Prediction Proceedings of the 5th WSEAS Int. Conf. on COMPUTATIONAL INTELLIGENCE, MANMACHINE SYSTEMS AND CYBERNETICS, Venice, Italy, November 20-22, 2006217

[14] Saxena, N. Hotchandani, Y. Kulshrestha, M. Arora, P. (2010). Stock Market Expert: Analyzing Stock Prices Proceedings of the 4th National Conference; INDIACom-2010 Computing For Nation Development, February 25 - 26, 2010 Bharati Vidyapeeth's Institute of Computer Applications and Management, New Delhi

[15] Kasemsan, M.L. and Radeerom, M. (2011). Intelligence Trading System for Thai Stock Index Based on Fuzzy Logic and Neuro-fuzzy System. Proceedings of the World Congress on Engineering and Computer Science 2011, I. San Francisco, USA.

[16] Feng, H. M. and Chou, H. C. (2011). Evolutional RBFNs prediction systems generation in the applications of financial time series data, Expert Systems with Applications, 38, 8285-8292.

[17] Abiyev, R. H. Abiyev, V. H. (2012). Differential Evaluation Learning of Fuzzy Wavelet Neural Networks for Stock Price Prediction, Journal of Information and Computing Science, 7(2), 121-130.

[18] ElAaL, M. M. A., Selim, G. and Fakhr, W. (2012). Stock Market Trend Prediction Model for the Egyptian Stock Market Using Neural Networks and Fuzzy Logic. D.-S. Huang et al. (Eds.): ICIC 2011, LNBI 6840, 85-90.

[19] Jandaghi, G., Reza Tehrani, R. Hosseinpour, D. Gholipour, R. and Shadkam, S.A.S., (2012)Application of Fuzzy-neural networks in multi-ahead forecast of stock price, African Journal of Business Management 4(6), 903-914.

[20] Prasanna, S. and Ezhilmaran, D. (2013), An analysis on Stock Market Prediction using Data Mining Techniques. International Journal of Computer Science \& Engineering Technology (IJCSET), 4(3), 49-51.

[21] Umoh, U. A, Nwachukwu, E. O.and Obot, O. U. (2010). Fuzzy Rule-based Framework for Effective Control of Profitability in a Paper Recycling Plant. Global Journal of Computer Science and Technology (GJCST), 10, 5667. 
[22] Umoh, U. A, Nwachukwu, E. O. and Okure, O. U. (2011). "Fuzzy-Neural Network Model for Effective Control of Profitability in a Paper Recycling Plant". American Journal of Scientific and Industrial Research (AJSIR). 2(4): 552-558.

[23] Pantazopoulos, K. N., Tsoukalas, L.H., Bourbakis, N. G., Brun, M. J. and Houstis, E. N. (1998). Financial prediction and trading strategies using neurofuzzy approaches. IEEE Transactions on Systems, Man and Cybernetics, Part B, 28(4), 520-531. Publishing Company, Boston.
[24] Takagi, T. and M. Sugeno, "Fuzzy identification of systems and its applications to modeling and control," IEEE Trans. Systems, Man, and Cybernetics, 15, 116$132,1985$.

[25] Guney, K. (2009). Comparison of Mamdani And Sugeno Fuzzy Inference System Models For Resonant Frequency Calculation Of Rectangular Microstrip Antennas. Progress In Electromagnetics Research B, 12, 81-104.

[26] Achelis, S. B. (2000). Technical Analysis from A to Z, Library of Congress Cataloging- in-Publication Data, ISBN 0-07-136348-3. McGraw Hill. 